\title{
Dispersibility of freeze-drying unmodified and modified TEMPO-oxidized cellulose nanofibrils in organic solvents
}

\author{
A. A. Luginina ${ }^{1}$, S. V. Kuznetsov ${ }^{1}$, V. K. Ivanov ${ }^{2}$, V. V. Voronov ${ }^{1}$, A. D. Yapryntsev ${ }^{2}$, D. I. Petukhov ${ }^{3}$, \\ S. Yu. Kottsov ${ }^{2}$, E. V. Chernova ${ }^{1}$, P. P. Fedorov ${ }^{1 *}$ \\ ${ }^{1}$ Prokhorov General Physics Institute of the Russian Academy of Sciences, \\ 38 Vavilov Street, Moscow 119991, Russia \\ ${ }^{2}$ Kurnakov Institute of General and Inorganic Chemistry of the Russian Academy of Sciences, \\ 31 Leninsky Prospect, Moscow 119991, Russia \\ ${ }^{3}$ Lomonosov Moscow State University, Chemical department, 1-3 Leninskie Gory, Moscow, 119991, Russia \\ *ppfedorov@yandex.ru
}

DOI 10.17586/2220-8054-2021-12-6-763-772

Cellulose nanofibrils (TOCNF) with a width of $20 \pm 6 \mathrm{~nm}$ and a length of $809 \pm 98 \mathrm{~nm}$ were prepared using 2,2,6,6-tetramethylpiperidinyl-1oxyl (TEMPO)-mediated oxidation. Two modifying agents were used to functionalize the TOCNF surface in aqueous media: alkyl ketene dimer (AKD) and 3-aminopropyltriethoxysilane (APS). The hydrophilic aerogel L-TOCNF, hydrophobic aerogels L-TOCNF-AKD and L-TOCNF-APS with water contact angles of $0,139 \pm 2$, and $133 \pm 2^{\circ}$, respectively, were prepared by freeze-drying of the aqueous dispersions. The elemental composition, morphology, sizes and crystal structure were determined by EDX analysis, scanning electron microscopy and X-ray diffraction, respectively. The process of redispersion of lyophilized samples in water and four organic solvents was investigated. The effect of TOCNF modification and solvent polarity on the redispersibility of lyophilized samples was revealed: the dispersibility of hydrophobic L-TOCNF-AKD and L-TOCNF-APS in organic solvents was significantly improved.

Keywords: TEMPO-oxidized cellulose nanofibrils, hydrophobic modification, freeze-drying, redispersibility.

Received: 25 October 2021

\section{Introduction}

Nanocellulose is one of the most promising environmentally friendly materials for a number of reasons, including: abundance of renewable sources for its production, biodegradability, biocompatibility, and non-toxicity [1]. Cellulosic nanomaterials have received increased attention in recent years due to their exceptional properties and potential for a variety of applications, including biomedical, energy storage, electronic devices, packaging, and composite materials [2-7], as well as the development of economical and green manufacturing methods. One such environmentally friendly method is TEMPO-mediated oxidation of cellulose pulp followed by mild mechanical disintegration treatment. This method allowed effective individualization of cellulose fibrils because of the formation of TEMPOoxidized cellulose nanofibrils (TOCNFs), which have uniform width and high aspect ratio (about 3 - $10 \mathrm{~nm}$ width and about $1 \mu \mathrm{m}$ length) with minimal energy consumption [8,9]. Single TOCNF elements have high mechanical strength (about $3 \mathrm{GPa}$ tensile strength and about $140 \mathrm{GPa}$ Young's modulus), low density of about $1.6 \mathrm{~g} / \mathrm{cm}^{3}$ and increased chemical reactivity, that are crucial for the development of new functional materials [10-12]. The hydrophilic nature of TOCNF requires its production as an aqueous dispersion. This creates serious transportation problems that can be avoided by drying. Drying also inhibits the growth of bacteria and fungi in nanocellulose [13]. TOCNFs, initially nanodispersed in water, form large agglomerates when aqueous dispersions are dried by natural evaporation, which leads to a loss of aforementioned TOCNF inherent functional properties [10]. Potential applications of TOCNF include their use as nanofillers in polymer composites. Most synthetic polymers are hydrophobic and, therefore, it is difficult to reinforce them with hydrophilic TOCNFs without aggregation the latter. As a result, preparation of the polymer composites requires mixing TOCNFs with polymer solutions in organic solvents.

One of the ways to obtain organic sols is the solvent gradual replacement. Isogai et al. [14] demonstrated the dispersibility of TOCNF in the various polar organic solvents such as dimethyl sulfoxide (DMSO), N,N-dimethylformamide (DMF), 1,3-dimethyl-2-imidazolidinone, N,N-dimethylacetamide (DMA) and 1-methyl-2-pyrrolidinone (NMP) by replacing -COONa groups with - $\mathrm{COOH}$ groups in TOCNF and subsequent solvent exchange. It was noted that the sodium salt form of TOCNFs could be dispersed only in DMSO. To solve the problem of dispersing TOCNF in the other organic solvents, various methods of surface modification, followed by solvent replacement, have been studied: 
- poly(ethylene glycol) (PEG)-grafted TOCNF-COO were individually dispersed in chloroform, toluene, dichloromethane, 1,4-dioxane, DMA [15-17];

- n-dodecylamine-modified TOCNF-COO were dispersed at the level of individual nanofibrils in isopropyl alcohol [18];

— cetyltrimethylammonium bromide-modified TOCNFs demonstrated increased hydrophobicity and improved redispersibility in DMF [19];

- TOCNFs, modified with tannic acid and hexadecylamine, were dispersed in toluene, ethanol, acetone [20];

- TOCNF-COO with amphiphilic diblock copolymer-modified surface exhibited an increased dispersibility in such organic solvents as DMSO, DMF, ethanol, and methanol [21].

It should be noted that the aforementioned solvent exchange process is quite laborious because of the use of intermediate solvents and multiple centrifugation cycles.

An alternative method to improve the redispersion of TOCNF includes the use of freeze-drying. Numerous studies were devoted to redispersion of modified and unmodified nanocrystals (CNC) and nanofibrils (CNF) of cellulose after the freeze-drying [22-30]. It is worth mentioning that TOCNFs have a large number of sodium carboxylate groups on nanofibril surfaces and much higher aspect ratios than CNC, so TOCNF cannot be redispersed in organic solvents using protocols previously developed for CNC.

Despite the urgency of the aforementioned problem, a relatively small number of papers have been devoted to the redispersion of lyophilized TOCNF in organic solvents. Jiang et al. [31] investigated the redispersion of lyophilized TOCNF as function of the medium composition and/or freezing conditions and found that TOCNFs, lyophilized from an aqueous dispersion, were redispersed only in water, while TOCNFs, lyophilized from tert-butanol/water mixtures, were readily dispersed in both water and DMF. Isogai $[10,11]$ confirmed that TOCNFs, lyophilized from tert-butanol/water mixtures, were readily dispersed in DMF. Indarti et al. [32] redispersed TOCNFs, thoroughly dried by freeze-drying, in chloroform. It is necessary to note the very low concentration of redispersed TOCNF $(0.5 \mathrm{mg}$ in $10 \mathrm{ml}$ of chloroform) and the short-term stability of the obtained dispersions (15 min) [32]. Araki et al. [33] showed that lyophilized PEG-grafted TOCNFs can be redispersed in water as well as in the non-polar solvent chloroform $(0.2 \%)$ by sonication. Johnson et al. [34] investigated the effect of hydrophobization of TOCNF by surface modification with octadecylamine on dispersion in organic solvents with a wide range of polarities and found that lyophilized hydrophobic TOCNFs could be dispersed by ultrasonic treatment in both polar and non-polar organic solvents (isopropyl alcohol, tetrahydrofuran and toluene). Chu et al. [35] revealed that the modification of TOCNFs and imparting hydrophobic properties to them were an effective way to improve the dispersion of TOCNF in organic solvents.

In this work, two modifying agents - alkyl ketene dimer (AKD) and 3-aminopropyltriethoxysilane (APS) were utilized in order to increase the hydrophobicity of TOCNF. This study evaluated the effect of the TOCNF modification and lyophilization on the redispersion ability in polar and non-polar solvents (DMSO, DMF, chloroform, hexane) as factors for increase in TOCNF redispersibility.

\section{Experimental section}

\subsection{Chemicals}

Ash free filter paper (FP) "Blue Ribbon" ( $I \beta$ crystal structure, crystallinity index 68.7, degree of polymerization 1112, particle width $25-50 \mu \mathrm{m}$, particle length $200-1500 \mu \mathrm{m}$ [36]); TEMPO (99\%) (NIOCH SB RAS, Russia); 3-aminopropyltriethoxysilane (97 \%) (PENTA-91 company, Russia); water emulsion of alkyl ketene dimer (mixture of stearic and palmitic acids, $60-40 \%$ by weight), (AKD) Flouise-200 brand containing $19 \%$ AKD and $3 \%$ anionic starch (Skiff Special Chemicals, Russia); 19 wt. \% sodium hypochlorite solution; sodium bromide; sodium hydroxide; acetic acid and hydrochloric acid (analytical grade); bidistilled water; and regenerated cellulose dialysis tube $12-14 \mathrm{kDa}$ molecular weight cut-off by Orange Scientific (Belgium) were used as starting materials. The solvents dimethyl sulfoxide (DMSO), dimethylformamide (DMF), chloroform, and hexane, chemically pure, (Chimmed, Russia) were used as received without additional purification.

\subsection{Preparation of the aqueous dispersion of TOCNF}

TOCNF was prepared by hydrolysis of FP with a $5.5 \mathrm{M}$ solution $\mathrm{HCl}$ at $80{ }^{\circ} \mathrm{C}$, using the TEMPO/ $\mathrm{NaBr} / \mathrm{NaClO}$ system in water at $\mathrm{pH} 10.0-10.5$, followed by ultrasonic treatment by the previously reported technique [37]. TOCNF yield was about $70 \%$. TOCNF dispersion was stored at $4{ }^{\circ} \mathrm{C}$. TOCNF content in the dispersion was determined gravimetrically. 


\subsection{Freeze-drying of the aqueous dispersion of TOCNF}

Aqueous TOCNF (1 wt.\%) dispersions $(100 \mathrm{~mL})$ in polypropylene containers were quickly frozen by immersing in liquid nitrogen $\left(-196{ }^{\circ} \mathrm{C}\right)$ for $10 \mathrm{~min}$, then lyophilized $\left(-5^{\circ} \mathrm{C}, 15 \div 20 \mathrm{~Pa}\right)$ for $48 \mathrm{~h}$ in a freeze-drier LS-1000 (Russia), followed by a rise in temperature up to $35{ }^{\circ} \mathrm{C}$ for 22 hours. The prepared sample was designated as LTOCNF.

\subsection{Preparation of freeze-dried modified TOCNF with AKD or APS}

The modification protocol of TOCNF with AKD is based on our previous research [38]. A certain amount $(0.1364 \mathrm{~g})$ of AKD aqueous emulsion was added to $1.00 \mathrm{wt} \%$ TOCNF dispersion $(100 \mathrm{~g})$ and sonicated for $5 \mathrm{~min}$ in an ice bath to obtain a sample loaded with $30 \mathrm{mg}$ dry AKD per gram dried TOCNF. After sonication, aqueous TOCNF-AKD mixtures were freeze-dried as described for aqueous dispersion of TOCNF.

The modification protocol of TOCNF with APS is based on our previous research [37]. The pH of bidistilled water was adjusted to 4.0 by dropping glacial acetic acid. APS aqueous solution (3.0 wt.\%) were prepared by adding $1.1068 \mathrm{~g}$ APS to $35.7865 \mathrm{~g}$ bidistilled water with $\mathrm{pH}=4.0$ under vigorous stirring in fluoroplastic beaker for $10 \mathrm{~min}$ while maintaining $\mathrm{pH}$ value at $\mathrm{pH}=4.0$. Then, $100 \mathrm{~g}$ of TOCNF dispersion $(1.38 \mathrm{wt} . \%)$ were added dropwise to APS solution and stirred with magnetic stirrer at room temperature for $30 \mathrm{~min}$, while maintaining a pH of 4.0. The $\mathrm{pH}$ value was adjusted using glacial acetic acid. The APS loading was $5 \mathrm{mmol}$ per gram dried TOCNF in the obtained sample. After stirring, aqueous TOCNF-APS mixtures were freeze-dried as described for aqueous dispersion of TOCNF.

The prepared samples were designated as L-TOCNF-AKD and L-TOCNF-APS. Freeze-dried samples were stored in a desiccator over phosphorus pentoxide.

\subsection{Redispersion of the L-TOCNF, L-TOCNF-AKD and L-TOCNF-APS in various solvents}

Water or an organic solvent (DMSO, DMF, chloroform or hexane) was added to the freeze-dried product of LTOCNF, L-TOCNF-AKD or L-TOCNF-APS to prepare $0.2 \%(\mathrm{w} / \mathrm{v})$ of nanofibrils. A weight of the freeze-dried sample (M) was taken with an accuracy of $0.0002 \mathrm{~g}$. After the freeze-dried samples were transferred to the solvent, the mixture was sonicated for $7 \mathrm{~min}$ (ultrasonic homogenizer with a $7 \mathrm{~mm}$ probe-tip diameter UZG13-01/22, 110 W, VNIITVCH, Russia) under ice bath cooling while maintaining a constant solvent volume. The concentration of each homogenized dispersion was maintained at $0.2 \%(\mathrm{w} / \mathrm{v})$.

The colloidal stability of the dispersions both after 1 hour and after 5 days was assessed visually according to the sedimentation and clarification of the dispersion. The dispersing degree $(D r)$ was calculated from the dry weight of the lyophilized product present in the supernatan and was used as a criteria for redispersibility. $D r$ was determined after ultrasonic treatment of the suspensions and their centrifugation at $11000 \mathrm{rpm}$ for $10 \mathrm{~min}$. Centrifugation was used to separate individualized nanofibrils from aggregates of nanofibrils. The precipitate and transparent supernatant were separated by pipetting. Solvents such as chloroform and hexane were removed from the supernatant by air drying at $35-40{ }^{\circ} \mathrm{C}$. Solvents (DMSO and DMF) were removed from the supernatant by drying in vacuum at $50{ }^{\circ} \mathrm{C}$ for $2 \mathrm{~h}$. The dried fraction of the lyophilized product present in the supernatant $\left(m_{s}, \mathrm{~g}\right)$ was weighed. $\operatorname{Dr}(\%)$ was determined as:

$$
D r=\frac{m_{s} \times 100}{M}
$$

where $M(\mathrm{~g})$ is the total dry weight of redispersed nanocellulose.

\subsection{Characterization}

X-ray diffraction patterns were recorded with Bruker D8 Advance diffractometer (Bruker AXS GmbH, Karlsruhe, Germany; $\mathrm{CuK} \alpha$-radiation; $8-60^{\circ} 2 \theta$ range, $0.02^{\circ} 2 \theta$ step). The $22.7^{\circ} 2 \theta$ diffraction peak was used for TOCNF crystallinity index (IC, \%) calculation by Segal's method [7]. X-ray diffraction data were obtained in reflection geometry, in which the incident radiation is most intensively scattered by crystallites whose reflecting planes are parallel to the axis of the fibrils, which makes it possible to determine their thickness. The transverse size of crystallites (D[200], $\mathrm{nm})$ was estimated using the Scherrer equation [36].

The degree of polymerization $(D P)$ was assessed by viscosity measurements using diluted solutions of dry TOCNF films in Cadoxen (cadmium ethylenediamine) [29]. DP was determined as an average of two independent measurements.

The carboxylate content ( $\mathrm{mmol} / \mathrm{g}$ ) in TOCNF was determined by a conductometric titration technique according to the protocol described elsewhere [39]. The conductivity values were recorded at room temperature using an Expert002 conductometer as the mean value of three measurements. 
TABLE 1. Characteristics of TOCNF

\begin{tabular}{|c|c|c|c|c|c|c|c|}
\hline \multicolumn{2}{|c|}{$\begin{array}{l}\text { Particle size } \\
(\mathrm{SEM}), \mathrm{nm}\end{array}$} & \multirow{2}{*}{$\begin{array}{c}\text { Particle } \\
\text { width } \\
\text { (AFM), nm }\end{array}$} & \multirow[t]{2}{*}{$D P$} & \multirow[t]{2}{*}{$\begin{array}{c}\zeta \text {-potential, } \\
\mathrm{mV}\end{array}$} & \multirow[t]{2}{*}{$\begin{array}{l}\text { Carboxyl group } \\
\text { content, } \mathrm{mmol} / \mathrm{g}\end{array}$} & \multirow[t]{2}{*}{ IC, $\%$} & \multirow[t]{2}{*}{$\mathrm{D}[200], \mathrm{nm}$} \\
\hline width & length & & & & & & \\
\hline $20 \pm 6$ & $809 \pm 98$ & $16 \pm 6$ & $123 \pm 3$ & $-50 \pm 2$ & $1.20 \pm 0.05$ & 78.2 & 2.9 \\
\hline
\end{tabular}

Zeta potential ( $\zeta$-potential) values of the dispersions were measured by an electrophoretic light scattering technique using the phase analysis light scattering (PALS) on the Photocor Compact- $Z$ analyzer at room temperature. The resulting $\zeta$-potential values are mean values in five consecutive measurements.

The morphology of the freeze-dried samples and the distribution of the elements on the surface were analyzed by simultaneous use of scanning electron microscopy (SEM) (NVision 40 microscope, Carl Zeiss NTS GmbH, Germany) and energy-dispersive X-ray spectroscopy (EDX), (X-Max detector, Oxford Instruments, UK). The size, and shape of nanofibrils were also analyzed by SEM. A drop of dilute aqueous dispersion of $0.01 \mathrm{wt} . \%$ TOCNF was applied onto a single crystal silicon substrate and air-dried for the SEM farther analysis. The size of nanofibrils, and pore size were revealed by the ImageJ software as a mean value in 25 independent measurements.

The surface morphology of TOCNF film was investigated using NTEGRA Prima atomic force microscope (NTMDT Spectrum Instruments, Russia) in a tapping mode. All experiments were carried out under controlled conditions maintained by a TRACKPORE ROOM-05 measuring complex (purity class 5 ISO (100), the accuracy of maintaining air temperature in the range of $23 \pm 5 \mathrm{~K}$ is $\pm 0.05 \mathrm{~K}$, the relative air humidity is $55 \pm 1 \%$ ).

The value of the specific surface area $\left(S_{B E T}\right)$ of the samples was measured by low-temperature adsorption of nitrogen using an ATKh-06 analyzer (KATAKON, Russia) with preliminary degassed stage for $1 \mathrm{~h}$ at $90{ }^{\circ} \mathrm{C}$ under vacuum. The specific surface area was calculated using the Brunauer-Emmett-Teller model (BET) and the five points method within the partial pressure range $0.05-0.25$.

The water contact angle (WCA) was measured on a FTA1000 Drop Shape Instrument B Frame System. The test sample was placed on a horizontal holder. The water droplet volume $100 \mu \mathrm{l}$ was applied onto the surface of the test sample by a special microdosing syringe. The images were recorded $1 \mathrm{~s}$ after application of the droplets using a $640 \times 480$ pixel CCD detector. The measurements were performed at room temperature $\left(24 \pm 2{ }^{\circ} \mathrm{C}\right)$ and repeated for 5 times on various fresh surfaces.

\section{Results and discussion}

\subsection{Characterization of TOCNF}

The characteristics of the TOCNF are summarized in Table 1. SEM images of the TOCNF samples show entangled nanofibrils with width $(20 \pm 6 \mathrm{~nm})$, and length $(809 \pm 98 \mathrm{~nm})$, Fig. 1(a). The fibril width according to AFM data is slightly lower, $16 \pm 6 \mathrm{~nm}$, Fig. 1(b). $\mathrm{HCl}$ hydrolysis followed by TEMPO oxidation significantly reduced the particle size (by three orders of magnitude) while also lowering the TOCNF degree of polymerization down to $123 \pm 3$ compared to the original FP cellulose (one order of magnitude), Table 1 [36].

The aqueous dispersions of TOCNF are stable for a long time (up to 6 months) due to the electrostatic repulsion of the carboxyl groups with content of $1.20 \pm 0.05 \mathrm{mmol} / \mathrm{g}$ TOCNF (Table 1 . The $\zeta$-potential value of TOCNF aqueous dispersions was $-50 \pm 2 \mathrm{mV}$ ).

X-ray diffraction pattern of TOCNF film (Fig. 2(a)) shows diffraction peak at $2 \theta=22.5^{\circ}$ and double peak in the range $2 \theta=14.8-16.8^{\circ}$ corresponding to the crystallographic planes (200), (11̄0) and (110) of monoclinic $I \beta$ cellulose [39]. The crystallinity $(78.2 \%)$ and a crystallite size (2.9 nm in the crystallographic plane [200]) are presented in Table 1.

\subsection{Morphology and physical properties of L-TOCNF, L-TOCNF-AKD, L-TOCNF-APS}

Freeze-drying yielded aerogels whose volume corresponded to the volume of the dispersion, Fig. 3(a-c). LTOCNF-APS aerogel has a pale yellow tint. For the samples after lyophilization, the X-ray diffraction pattern retains initial structure of $I \beta$ cellulose (Fig. 2(b-d)), but the intensity of diffuse halo increases. The crystallinity index from TOCNF film (78.2 \%) to $69.1 \%$ for L-TOCNF (Fig. 3d) was decreased.

The crystallinities of the modified L-TOCNF-AKD and L-TOCNF-APS were slightly decreased compared to LTOCNF, due to the introduction of amorphous AKD or APS on the surface of the cellulose nanofibrils, Fig. 3(d). However, the crystallite size D[200] in the L-TOCNF-AKD and L-TOCNF-APS samples no showed obvious changes after modification (Fig. 3(d)). This occurred due to the modification that occurred only on the surface of the cellulose 


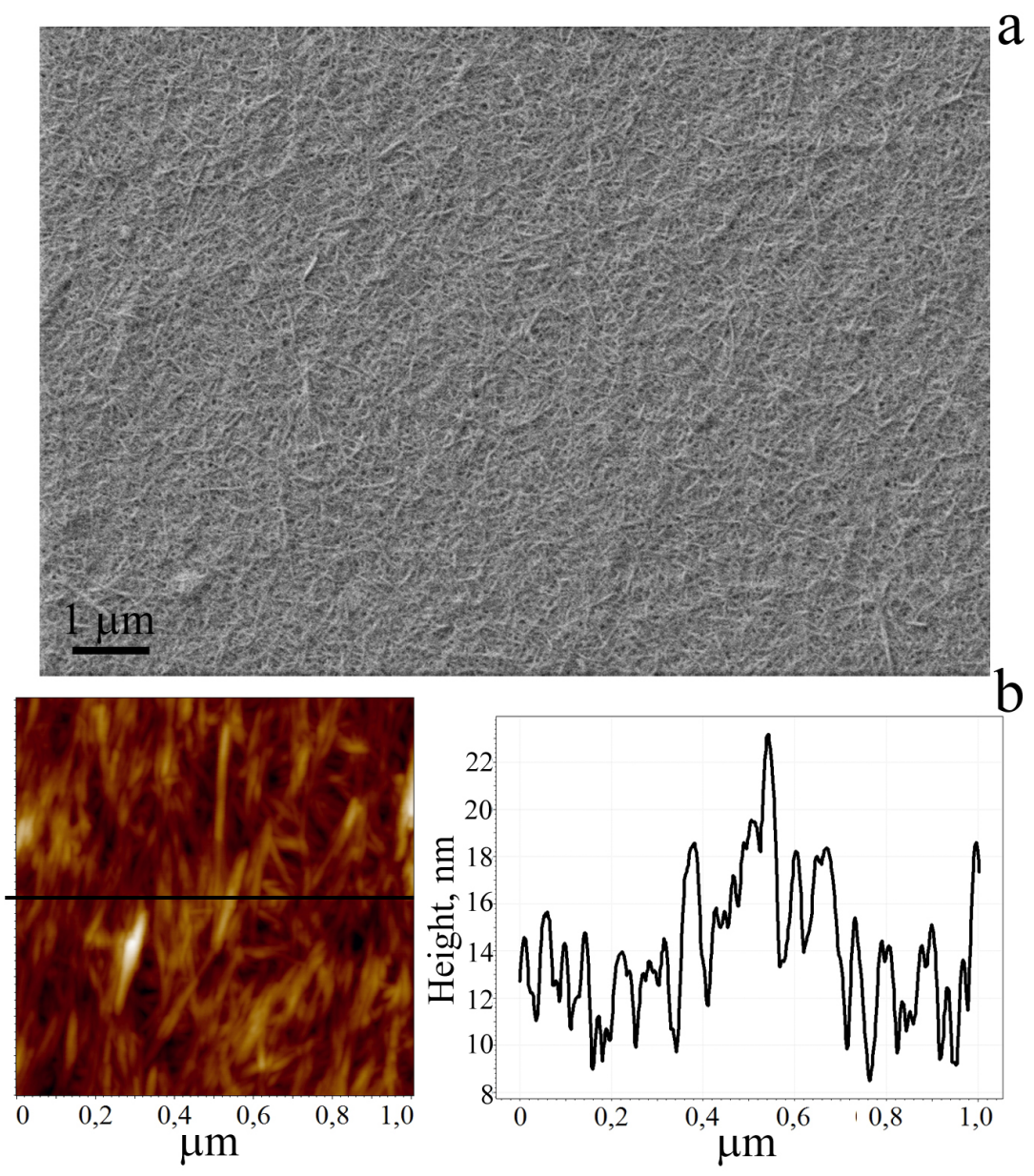

FIG. 1. SEM images of diluted dispersions of TOCNF $0.01 \mathrm{wt} \%$, dried on a single-crystal silicon substrate (a), AFM images of TOCNF film and height profile along the black line (b)

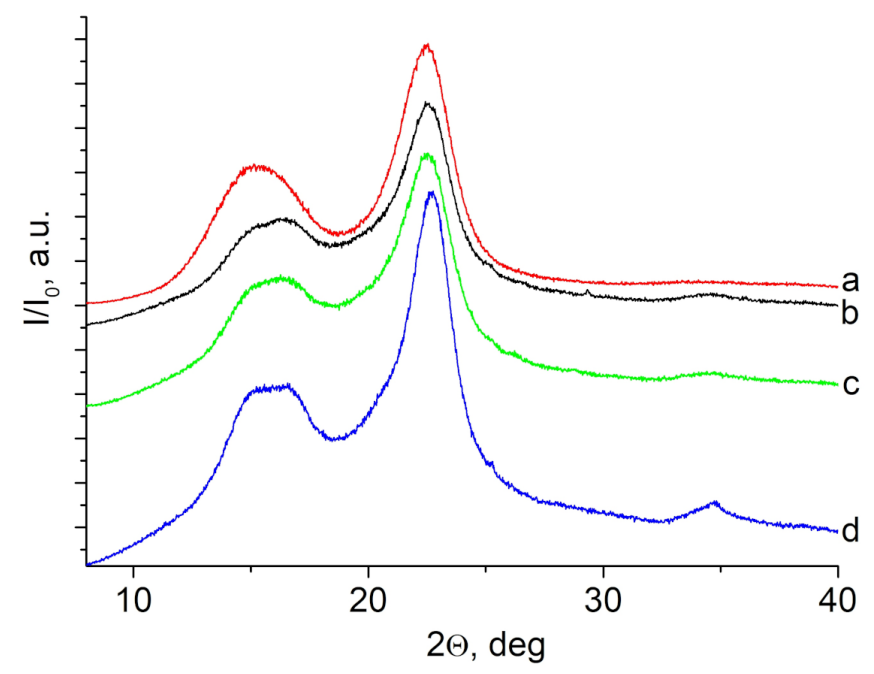

FIG. 2. XRD patterns of the samples: TOCNF (a), L-TOCNF (b), L-TOCNF-AKD (c), L-TOCNF-APS (d) 


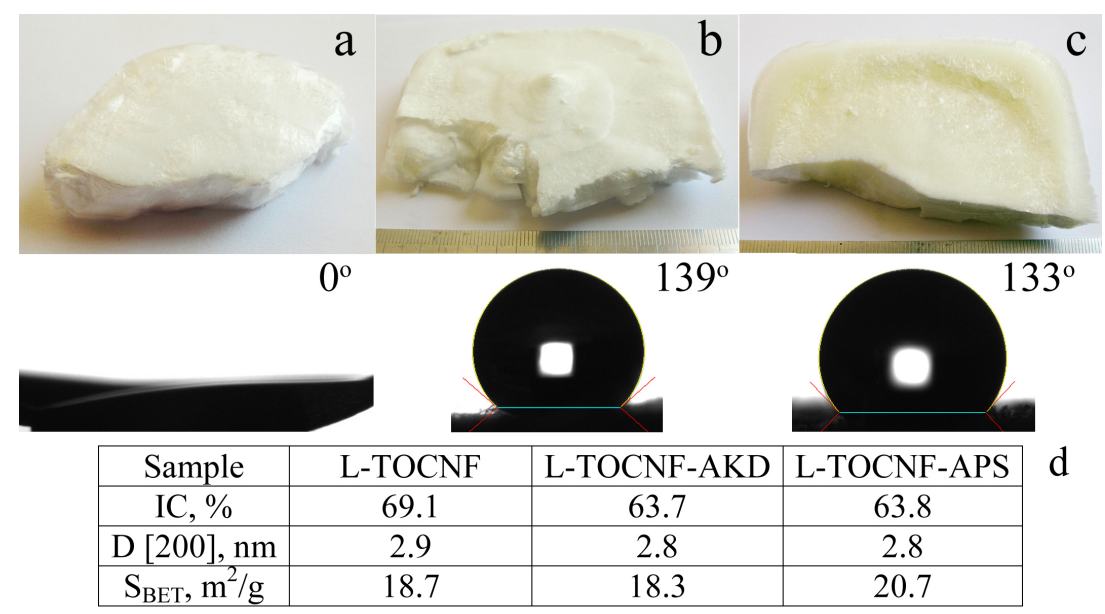

FIG. 3. Photographic images of aerogels and water drops (WCA) on the surface L-TOCNF (a), L-TOCNF-AKD (b), L-TOCNF-APS(c), and their physical properties (d)

nanofibrils with the simultaneous increase of the proportion of the amorphous phase, and retained the crystalline structure of the cellulose. The BET specific surface areas were in the range of $18.7-20.7 \mathrm{~m}^{2} / \mathrm{g}$ for all aerogels (Fig. 3(d)).

The morphology of the lyophilized samples is characterized by scanning electron microscopy. TOCNFs form layered porous aerogels with ribbon-like film structures (Fig. 4(a)) which are the result of the lateral agglomeration of cellulose nanofibrils. Similar morphology was observed by Han et al. and Du et al. [25,40], which explained by mean of lateral aggregation due to hydrogen bonding.

After lyophilization of L-TOCNF-AKD and L-TOCNF-APS hydrosols, porous plate-like materials were obtained (Fig. 4(b,c)). Aerogels contain heterogeneously shaped pores with a wide range of sizes from four to several tens micrometers. The thickness of the L-TOCNF, L-TOCNF-AKD, and L-TOCNF-APS layers were estimated from SEM photographs as $15-30,30-60$, and $45-80 \mathrm{~nm}$, respectively. The increase in the thickness of the L-TOCNF-AKD and L-TOCNF-APS layers is apparently associated with surface modification $[37,38]$. EDX results showed an increased $\mathrm{C}$ content and a lower $\mathrm{O}$ content in L-TOCNF-AKD sample compared to the L-TOCNF sample, which confirms the presence of aliphatic chains of fatty acids (stearic and palmitic) on the surface of L-TOCNF-AKD (Fig. 4(d,e)). The hydrophobic nature of AKD-modified TOCNFs is due to the adsorption through electrostatic interaction of AKD with dissociated carboxyl groups of TOCNF, as well as the formation of $\beta$-ketoester bonds between AKD and TOCNF [38]. EDX analysis indicated the presence of $N=5.7 \pm 0.4$ at. $\%$ and $S i=5.6 \pm 0.4$ at. $\%$ in L-TOCNF-APS sample due to the coating of its surface with aminopolysiloxane networks, Fig. 4(f), as shown in our previous study [37].

Unmodified L-TOCNF exhibited hydrophilic properties as the aerogel immediately absorbed a drop of water $\left(\mathrm{WCA}=0^{\circ}\right)$. In contrast, the modified L-TOCNF-AKD (WCA $139 \pm 2^{\circ}$ ) and L-TOCNF-APS $\left(\right.$ WCA $133 \pm 2^{\circ}$ ) were highly hydrophobic (Fig. 3(a-c)).

\subsection{Redispersing of lyophilized L-TOCNF, L-TOCNF-AKD and L-TOCNF-APS}

The main purpose of hydrophobizing TOCNFs is to improve their dispersibility in organic solvents. Solvents are usually classified according to their dissolving ability as polar and nonpolar. However, the polarity of a solvent cannot be expressed in a specific physical quantity. Dielectric constant $(\varepsilon)$ and dipole moment $(\mu)$ are often used to quantify the polarity of a solvent [41]. Hexane $(\varepsilon=1.9, \mu=0)$, chloroform $(\varepsilon=4.7, \mu=1.15)$, DMF $(\varepsilon=36.7, \mu=3.2)$ and DMSO ( $\varepsilon=46.7, \mu=4.0$ ) were selected as nonpolar organic solvent, low and medium polarity solvents, respectively. For comparison, all lyophilized samples were redispersed in high polarity solvent - water $(\varepsilon=78.5$, $\mu=1.8)$.

Suspensions of the lyophilized samples were sonicated to disrupt aggregation between nanofibers after freezedrying. The lyophilized unmodified and modified TOCNFs were redispersed in various solvents, presented in Fig. 5. The fact that sonication is effective for homogenizing dispersions has been also demonstrated in previous studies [42, 43]. The need for sonication indicates the endothermic nature of the redispersion. Both after 1 hour and after 5 days aging, the sedimentation and clarification of the suspension were checked. L-TOCNF showed excellent redispersibility to transparent dispersions in water and dispersion stability after both 1 hour and 5 days (Fig. 5(a,b)). The resulting clear stable dispersion and high dispersing degree $(D r=98 \%)$ of L-TOCNF indicate successful nanofibrillation of lyophilized TOCNF in water. L-TOCNF was also redispersed in DMSO with formation of a translucent dispersion 


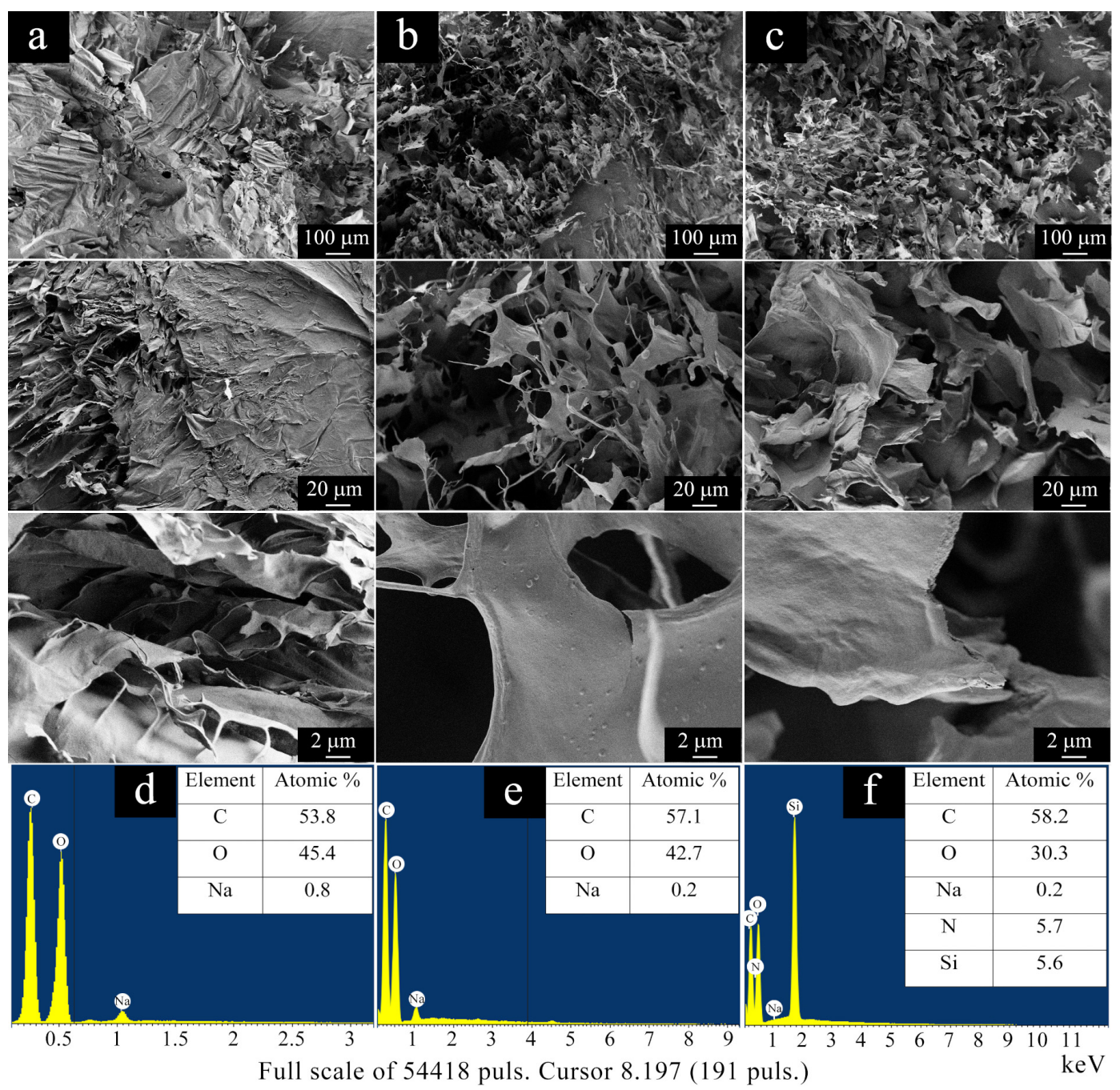

FIG. 4. SEM images and EDX of the samples: L-TOCNF (a, d), L-TOCNF-AKD (b, e), L-TOCNFAPS $(c, f)$

after 1 hour and little sedimentation after 5 days (Fig. 5(a,b)) and $D r=67 \%$, (Fig. 6). L-TOCNF did not disperse in DMF with opaque dispersion (Fig. 5(a)) formation and sedimentation after 5 days (Fig. 5(b)) $(D r=5 \%)$. L-TOCNF did not disperse in chloroform and hexane due to their very low dielectric constant (Figs. 5, 6).

The hydrophobic L-TOCNF-AKD and L-TOCNF-APS has not shown sign of redispersability in water, as the particles started immediately separating after sonication. Even flotation was observed for L-TOCNF-AKD, due to the presence of long aliphatic chains fatty acid (stearic and palmitic) on the TOCNF surface (Fig. 5). On the contrary, sedimentation of L-TOCNF-APS in aqueous suspensions occurred despite its high wetting angle (WCA $133 \pm 2^{\circ}$ ) due to the presence of polar $\mathrm{NH}_{2}$ groups in the grafted polyaminopropylsiloxane (Fig. 5). L-TOCNF-AKD and LTOCNF-APS showed good redispersibility in medium polarity solvents such as DMSO, DMF, which is confirmed by translucent dispersions after 1 hour (Fig. 5(a)) and slight sedimentation after 5 days (Fig. 5(b)) and high $D r$ (Fig. 6). It should be noted that L-TOCNF-AKD $(D r=97 \%)$ is more readily redispersible in a less polar solvent (e.g., DMF) than L-TOCNF-APS ( $D r=75 \%)$. The dispersion of L-TOCNF-AKD no showed signs of sedimentation for 5 days (Fig. 5(b)). This effect can be attributed to the hydrophobic aliphatic chains of AKD, which prevent the formation of TOCNF intermolecular hydrogen bonds due to steric hindrance. L-TOCNF-AKD and L-TOCNF-APS was demonstrated partial redispersibility in the low-polarity solvent chloroform (Fig. 5), $\mathrm{Dr}=34 \%$ and $\mathrm{Dr}=11 \%$ respectively (Fig. 6). In this case, the same pattern is observed as in the case of redispersibility in DMF. However, neither L-TOCNF-AKD nor L-TOCNF-APS can be redispersed in the non-polar solvent hexane (Figs. 5, 6). Finally, the redispersibility of lyophilized modified TOCNFs in organic solvents decreases with decreasing solvent polarity up to the complete absence of redispersibility in a nonpolar solvent hexane. 

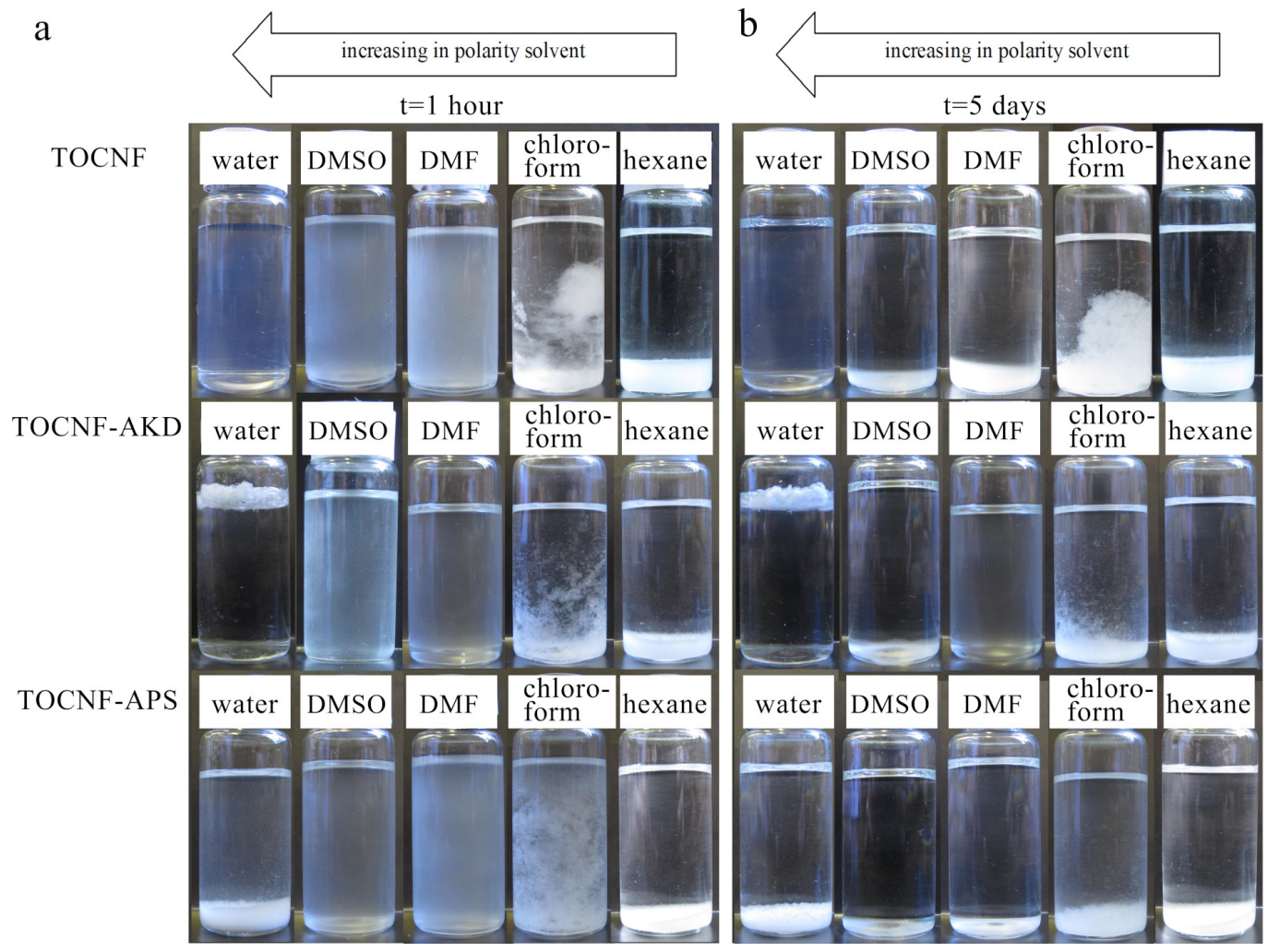

FIG. 5. Photograph of L-TOCNF, L-TOCNF-AKD, L-TOCNF-APS dispersed in different solvents $(0.2 \% \mathrm{w} / \mathrm{v})$ after 1 hour (a) and 5 days (b)

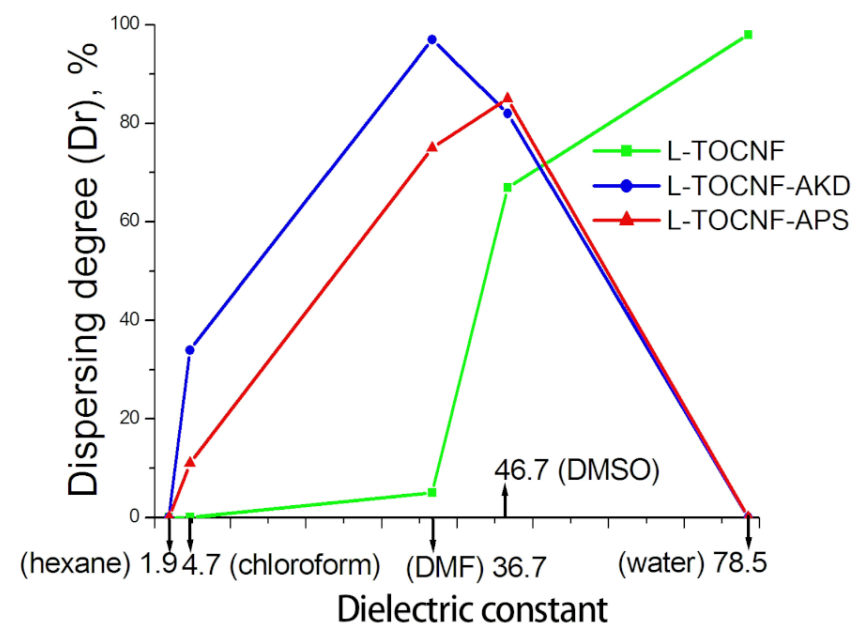

FIG. 6. Correlation between the dispersion degree in organic solvents L-TOCNF, L-TOCNF-AKD, L-TOCNF-APS and $\varepsilon$ solvent 


\section{Conclusions}

The hydrophobic aerogels L-TOCNF-AKD (WCA $139 \pm 2^{\circ}$ ) and L-TOCNF-APS (WCA $133 \pm 2^{\circ}$ ) were prepared by freeze-drying. The redispersion of lyophilized samples of unmodified and modified TOCNFs in organic solvents was studied by the method of visualization and assessment of the dispersing degree. It was shown that hydrophilic L-TOCNF samples are redispersed in water and in the polar solvent DMSO, but not in less polar and nonpolar organic solvents. The redispersibility of hydrophobic L-TOCNF-AKD and L-TOCNF-APS in organic solvents has been significantly improved. This may be due to the significantly reduced polarity of the modified chain on the surface. Transparent supernatant dispersions containing individualized nanofibrils were observed in DMSO and DMF. Partial redispersibility was noted in the low-polarity solvent (chloroform). None of the samples can be redispersed in a nonpolar solvent such as hexane. L-TOCNF-AKD redispersed better in less polar solvents such as DMF and chloroform than L-TOCNF-APS due to hydrophobic aliphatic AKD chains on the surface of TOCNF. The developed approach can be applied to improve of redispersibility of lyophilized modified TOCNFs in organic solvents without using laborious solvent replacing technique.

\section{Acknowledgements}

This work was supported by the Ministry of Science and Higher Education within the State assignment to Prokhorov General Physics Institute of the Russian Academy of Sciences. Authors express their sincere gratitude to Dr. Arthur I. Popov for his most kind assistance in the preparation of the present manuscript, Dr. Radmir V. Gainutdinov for the study of Atomic Force Microscopy, Dr. Khursand Yorov for freeze-drying and Dr. Vladimir N. Kuryakov for zeta potential measurements.

\section{References}

[1] De France K., Zeng Z., Wu T., Nyström G. Functional Materials from Nanocellulose: Utilizing Structure-Property Relationships in Bottom-Up Fabrication. Advanced Materials, 2021, 33 (28), P. 2000657.

[2] De Amorim J.D.P., de Souza K.C., et al. Plant and bacterial nanocellulose: production, properties and applications in medicine, food, cosmetics, electronics and engineering. A review. Environ. Chem. Lett., 2020, 18, P. 851-869.

[3] Liu Z., Zhang S., He B. et al. Synthesis of cellulose aerogels as promising carriers for drug delivery: a review. Cellulose, 2021, 28, P. 26972714.

[4] Ahankari S.S., Subhedar A.R., Bhadauria S.S., Dufresne A. Nanocellulose in food packaging: a review. Carbohyd. Polym., 2020, 255, P. 117479.

[5] Dias O.A.T., Konar S., et al. Current State of Applications of Nanocellulose in Flexible Energy and Electronic Devices. Front. Chem., 2020, 8, P. 420.

[6] Nie S., Hao N., et al. Cellulose nanofibrils-based thermally conductive composites for flexible electronics: a mini review. Cellulose, 2020, 27, P. 4173-4187.

[7] Luginina A.A., Kuznetsov S.V., et al. Hydrophobization of up-conversion luminescent films based on nanocellulose/MF 2 :Ho particles $(\mathrm{M}=$ Sr, Ca) by acrylic resin. Nanosystems: Phys. Chem. Math., 2019, 10 (5), P. 585-598.

[8] Saito T., Kimura S., Nishiyama Y., Isogai A. Cellulose nanofibers prepared by TEMPO-mediated oxidation of native cellulose. Biomacromolecules, 2007, 8 (8), P. 2485-2491.

[9] Isogai A., Saito T., Fukuzumi H. TEMPO-oxidized cellulose nanofibers. Nanoscale, 2011, 3 (1), P. 71-85.

[10] Isogai A. Development of completely dispersed cellulose nanofibers. Proceedings of the Japan Academy, Series B, 2018,94 (4), P. 161-179.

[11] Isogai A. Emerging Nanocellulose Technologies: Recent Developments. Advanced Materials, 2020, 53, P. 2000630.

[12] Forti E.S., El AwadAzrak S.M., et al. Mechanical enhancement of cellulose nanofibril (CNF) films through the addition of water-soluble polymers. Cellulose, 2021, 28 (10), P. 6449-6465.

[13] Beck S., Bouchard J., Berry R. Dispersibility in Water of Dried Nanocrystalline Cellulose. Biomacromolecules, 2012,13 (5), P. $1486-1494$.

[14] Okita Y., Fujisawa S., Saito T., Isogai A. TEMPO-Oxidized Cellulose Nanofibrils Dispersed in Organic Solvents. Biomacromolecules, 2011, 12, P. 518-522.

[15] Fujisawa S., Saito T., et al. Surface Engineering of Ultrafine Cellulose Nanofibrils toward Polymer Nanocomposite Materials. Biomacromolecules, 2013, 14 (5), P. 1541-1546.

[16] Fujisawa S., Saito T., et al. Comparison of mechanical reinforcement effects of surface-modified cellulose nanofibrils and carbon nanotubes in PLLA composites. Composites Science and Technology, 2014, 90, P. 96-101.

[17] Soeta H., Fujisawa S., Saito T., Isogai A. Controlling Miscibility of the Interphase in Polymer-Grafted Nanocellulose/Cellulose Triacetate Nanocomposites. ACS Omega, 2020, 5 (37), P. 23755-23761.

[18] Fujisawa S., Saito T., Isogai A. Nano-dispersion of TEMPO-oxidized cellulose/aliphatic amine salts in isopropyl alcohol. Cellulose, 2012, 19, P. 459-466.

[19] Qu J., Yuan Z., et al. Enhancing the redispersibility of TEMPO-mediated oxidized cellulose nanofibrils in N,N-dimethylformamide by modification with cetyltrimethylammonium bromide. Cellulose, 2019, 26, P. 7769-7780.

[20] Shrestha S., Chowdhury R.A., et al. Surface hydrophobization of TEMPO-oxidized cellulose nanofibrils (CNFs) using a facile, aqueous modification process and its effect on properties of epoxy nanocomposites. Cellulose, 2019, 26, P. 9631-9643.

[21] Dong H., Napadensky E., et al. Cellulose Nanofibrils and Diblock Copolymer Complex: Micelle Formation and Enhanced Dispersibility. ACS Sustain. Chem. Eng., 2016, 5 (2), P. 1264-1271.

[22] Viet D., Beck-Candanedo S., Gray D.G. Dispersion of cellulose nanocrystals in polar organic solvents. Cellulose, 2007,14, P. 109-113. 
[23] Heux L., Chauve G., Bonini C. Nonflocculating and Chiral-Nematic Self-ordering of Cellulose Microcrystals Suspensions in Nonpolar Solvents. Langmuir, 2000, 16 (21), P. 8210-8212.

[24] Peng S.X., Chang H., et al. A comparative guide to controlled hydrophobization of cellulose nanocrystals via surface esterification. Cellulose, 2016, 23 (3), P. 1825-1846.

[25] Han J., Zhou C., et al. Self-assembling behavior of cellulose nanoparticles during freeze-drying: effect of suspension concentration, particle size, crystal structure, and surface charge. Biomacromolecules, 2013, 14, P. 1529-1540.

[26] Kaboorani A., Riedl B., Surface modification of cellulose nanocrystals (CNC) by a cationic surfactant. Ind. Crops Prod., 2015, 65, P. 45-55.

[27] Samir M.A.S.A., Alloin F., et al. Preparation of Cellulose Whiskers Reinforced Nanocomposites from an Organic Medium Suspension. Macromolecules, 2004, 37 (4), P. 1386-1393.

[28] Tang L.M., Weder C. Cellulose Whisker/Epoxy Resin Nanocomposites. ACS Appl. Mater. Interfaces, 2010,2 (4), P. 1073-1080.

[29] Voronova M.I., Surov O.V., et al. Dispersibility of Nanocrystalline Cellulose in Organic Solvents. Russian Journal of Bioorganic Chemistry, 2020, 46 (7), P. 1295-1303.

[30] Hu Z., Berry R.M., Pelton R., Cranston E.D. One-Pot Water-Based Hydrophobic Surface Modification of Cellulose Nanocrystals Using Plant Polyphenols. ACS Sustain. Chem. Eng., 2017, 5, P. 5018-5026.

[31] Jiang F., Hsieh Y.L. Assembling and redispersibility of rice straw nanocellulose: effect of tert-butanol. ACS Appl. Mater. Interfaces, 2014, 6, P. 20075-20084.

[32] Indarti E., Marwan, Wanrosli W.D. Dispersion Stability of Nanocellulose in Nonpolar Solvent: Chloroform. Materials Science Forum, 2020, 998, P. 170-175.

[33] Araki J., Wada M., Kuga S. Steric Stabilization of a Cellulose Microcrystal Suspension by Poly(ethylene glycol) Grafting. Langmuir, 2001,17 (1), P. 21-27.

[34] Johnson R.K., Zink-Sharp A., Glasser W.G. Preparation and characterization of hydrophobic derivatives of TEMPO-oxidized nanocelluloses. Cellulose, 2011, 18, P. 1599-1609.

[35] Chu Y., Sun Y., Wu W., Xiao H. Dispersion Properties of Nanocellulose: A Review. Carbohyd. Polym., $2020,250$. P. 116892.

[36] Fedorov P.P., Luginina A.A., et al. Composite up-conversion luminescent ?lms containing a nanocellulose and $\mathrm{SrF}_{2}$ :Ho particle. Cellulose, 2019, 26 (4), P. 2403-2423.

[37] Luginina A.A., Kuznetsov S.V., et al. Laser damage threshold of hydrophobic up-conversion carboxylated nanocellulose/SrF 2 :Ho composite films functionalized with 3-aminopropyltriethoxysilane. Cellulose, 2021, 28, P. 10841-10862.

[38] Fedorov P.P., Luginina A.A., et al. Hydrophobic up-conversion carboxylated nanocellulose/fluoride phosphor composite films modified with alkyl ketene dimer. Carbohyd. Polym., 2020, 250, P. 116866.

[39] Jiang F., Han S., Hsieh Y.L. Controlled defibrillation of rice straw cellulose and selfassembly of cellulose nanofibrils into highly crystalline fibrous materials. RSC Advances, 2013, 3 (30), P. 12366-12375.

[40] Du L., Yu Z., Wang J., et al. Analyzing the film formation mechanism of cellulose nanoparticles (CNPs) based on the fast freeze-drying morphology. Cellulose, 2020, 27, P. 6921-6933.

[41] Reichardt C. Solvents and Solvent Effects in Organic Chemistry. Second ed., Mir Publishers, Moscow, 1991, 763 p.

[42] Kasuga T., Isobe N., et al. Clearly transparent nanopaper from highly concentrated cellulose nanofiber dispersion using dilution and sonication. Nanomaterials, 2018, 8 (2), P. 104.

[43] Sharma A., Mandal T., Goswami S. Dispersibility and Stability Studies of Cellulose Nanofibers: Implications for Nanocomposite Preparation. J. Polym. Environ., 2021, 29, P. 1516-1525. 